\title{
Cation-Mutation Design of Quaternary Nitride Semiconductors lattice-matched to $\mathrm{GaN}$
}

\author{
Zeng-Hua Cai ${ }^{1}$, Prineha Narang ${ }^{2}$, Harry A. Atwater ${ }^{2}$, Shiyou Chen ${ }^{*}{ }^{1}$, Chun-Gang Duan ${ }^{1}$, \\ Zi-Qiang $\mathrm{Zhu}^{1}$, and Jun-Hao $\mathrm{Chu}^{1}$ \\ ${ }^{1}$ Key Laboratory of Polar Materials and Devices (MOE), East China Normal University, Shanghai \\ ${ }^{2}$ Thomas J. Watson Laboratory of Applied Physics, California Institute of Technology, Pasadena, USA
} 200241, China

Table S1. Space group and formation energies $\Delta \mathrm{H}_{\mathrm{f}}$ (in eV/formula unit) of all the nitride secondary phases.

\begin{tabular}{|c|c|c|}
\hline & Space Group & $\Delta \mathbf{H}_{\mathbf{f}}$ \\
\hline $\mathrm{Ge}_{3} \mathrm{~N}_{4}$ & $P 31 c$ & -0.39 \\
\hline $\mathrm{Cu}_{3} \mathrm{~N}$ & $P m-3 m$ & -0.02 \\
\hline $\mathrm{CuN}_{3}$ & $I 4_{1} /$ amd & 1.887 \\
\hline $\mathrm{CuN}_{6}$ & Pmcn & 4.07 \\
\hline $\mathrm{CuN}$ & $F-43 m$ & 1.553 \\
\hline $\mathrm{GaN}$ & $P 6_{3} m c$ & -0.972 \\
\hline AlN & $P 6_{3} m c$ & -2.829 \\
\hline $\operatorname{InN}$ & $P 6_{3} m c$ & 0.158 \\
\hline $\mathrm{Li}_{3} \mathrm{~N}$ & $P 6 / \mathrm{mmm}$ & -1.48 \\
\hline $\mathrm{LiN}_{3}$ & $C 2 / m$ & -0.587 \\
\hline $\mathrm{NaN}_{3}$ & $R-3 m$ & -0.48 \\
\hline $\mathrm{Na}_{3} \mathrm{~N}$ & $P m-3 m$ & 0.897 \\
\hline $\mathrm{KN}_{3}$ & $\mathrm{I} / \mathrm{mcm}$ & -0.661 \\
\hline $\mathrm{K}_{3} \mathrm{~N}$ & $\mathrm{~Pb}_{3} / \mathrm{mcm}$ & 1.891 \\
\hline $\mathrm{AgN}_{3}$ & Ibam & 2.44 \\
\hline $\mathrm{Li}_{5} \mathrm{GeN}_{3}$ & $P 1$ & -4.453 \\
\hline $\mathrm{Li}_{3} \mathrm{GaN}_{2}$ & $I a-3$ & -3.232 \\
\hline $\mathrm{NaGe}_{2} \mathrm{~N}_{3}$ & $\mathrm{Cmc}_{1}$ & -1.326 \\
\hline $\mathrm{Li}_{3} \mathrm{AlN}_{2}$ & $I a-3$ & -5.128 \\
\hline $\mathrm{LiGe}_{2} \mathrm{~N}_{3}$ (never reported) & $\mathrm{Cmc2} 2_{1}$ & -2.054 \\
\hline
\end{tabular}


Table S2. Space group and formation energies $\Delta \mathrm{H}_{\mathrm{f}}$ (in $\mathrm{eV} /$ formula unit) of all the non-nitride secondary phases.

\begin{tabular}{|c|c|c|}
\hline & Space Group & $\Delta \mathbf{H}_{\mathbf{f}}(\mathbf{e V} / \mathbf{u n i t})$ \\
\hline $\mathrm{Li}_{9} \mathrm{Ge}_{4}$ & $\mathrm{Cmcm}$ & -4.522 \\
\hline $\mathrm{Li}_{3} \mathrm{Ge}$ & $F m-3 m$ & -1.311 \\
\hline $\mathrm{Li}_{15} \mathrm{Ge}_{4}$ & $I-43 d$ & -6.199 \\
\hline $\mathrm{Li}_{11} \mathrm{Ge}_{6}$ & Ccmm & -5.552 \\
\hline $\mathrm{Li}_{7} \mathrm{Ge}_{2}$ & $\mathrm{Cmmm}$ & -2.885 \\
\hline $\mathrm{LiGe}$ & $I 4_{1} / a$ & -0.567 \\
\hline $\mathrm{LiGa}$ & $F d-3 m$ & -0.649 \\
\hline $\mathrm{Li}_{3} \mathrm{Ga}_{2}$ & $R-3 m$ & -1.601 \\
\hline $\mathrm{Li}_{2} \mathrm{Ga}$ & $\mathrm{Cmcm}$ & -0.899 \\
\hline $\mathrm{Li}_{3} \mathrm{Ga}_{7}$ & $R-3 m$ & -2.129 \\
\hline $\mathrm{LiGa}_{3}$ & $P m-3 m$ & -0.732 \\
\hline $\mathrm{L}_{\mathrm{i} 3} \mathrm{Al}_{2}$ & $R-3 m$ & -0.907 \\
\hline LiAl & $F d-3 m$ & -0.359 \\
\hline $\mathrm{Li}_{9} \mathrm{Al}_{4}$ & $C 2 / m$ & -2.027 \\
\hline $\mathrm{LiAl}_{3}$ & $P m-3 m$ & -0.325 \\
\hline $\mathrm{Li}_{3} \operatorname{In}_{2}$ & $R-3 m$ & -1.513 \\
\hline $\mathrm{Li}_{2} \mathrm{In}$ & $\mathrm{Cmcm}$ & -0.889 \\
\hline LiIn & $F d-3 m$ & -0.535 \\
\hline $\mathrm{Li}_{3} \mathrm{In}$ & $F m-3 m$ & -0.988 \\
\hline $\mathrm{Li}_{13} \operatorname{In}_{3}$ & $F d-3 m$ & -3.368 \\
\hline $\operatorname{LiIn}_{3}$ & $P m-3 m$ & -0.56 \\
\hline LiGaGe & $\mathrm{Pb}_{3} m c$ & -0.806 \\
\hline LiAlGe & $F-43 m$ & -0.885 \\
\hline LiInGe & $F-43 m$ & -0.726 \\
\hline
\end{tabular}

